\title{
Developing and Deploying Universal Diagnostic Platforms for One-Health Biosurveillance
}

\author{
Harshini Mukundan* \\ Chemistry, Los ALamos National Laboratory, Los ALamos, NM, USA
}

\section{Objective}

Our goal is to develop deployable strategies for infectious disease diagnosis at the point-of-care that are applicable to multiple hosts of infection - conforming to the global One Health Strategy for diagnostics. We aim to develop methods that do not require prior knowledge of the pathogen in question, and can facilitate rapid and effective decision-making and situational awareness.

\section{Introduction}

There is an urgent need for diagnostic strategies for infections which are host-independent, so as to effectively track zoonotic spread, monitor animal carriers of pathogens, and evaluate transmission dynamics. Infection of a host - pathogen or human- by an animal results in recognition by the immune response, which consequently causes release of inflammatory mediators. Many scientists have explored the use of cytokines as diagnostic indicators of disease, but the conserved nature of the immune response in humans and animals results in cross-reactivity among many pathogens, making evaluation of the results difficult, especially in high disease burden populations. Measuring the pathogen-specific signature, however, is advantageous - as it offers discrete identification of active infection, and discrimination from exposure. It also offers a universal strategy that can be applied to human and animal hosts of infection - allowing for One Health Biosurveillance. Achieving this, however, requires the development of a) tailored strategies for the measurement of biochemically disparate pathogen signatures in clinical samples and b) ultra-sensitive detection of such signatures in the host. The sensor team at Los Alamos National Laboratory is working on both of these aspects, and the development of One Health Diagnostic platforms, the focus of the work presented here.

\section{Methods}

Many of the biomarkers secreted by bacterial pathogens and recognized by innate immune receptors elicit host cytokine responses that are amphiphilic (largely glycolipids, lipoproteins or lipoglycans). Based on such, our team has developed tailored methods - membrane insertion and lipoprotein capture for the capture and detection of these greasy and stealthy molecules in infected blood. Sensitivity and specificity, assay optimization, alinearity associated with lipidic molecules and assay parameter development for biomarkers associated with bacterial pathogens will be presented. Considerations for clinical sampling for amphiphile detection in blood, and clinical study design will be demonstrated. Our team has developed an ultra-sensitive biosensor for detection of biomarkers in complex matrices based on the evanescent field properties of single mode planar optical waveguides. Detection of amphiphilic biomarkers in clinical samples, using the waveguide-based biosensor and tailored methods for the detection of such molecules has allowed for the diagnosis of infection in both human and animals hosts. Herein, we present two examples of the same; 1) diagnosis of tuberculosis in humans, cattle and badgers using pathogen-based diagnostic strategies; and 2) detection of Gram-negative Lipopolysacharides in beef and in patients with Salmonella-induced sepsis.

\section{Results}

We have developed tailored ultra-sensitive waveguide-biosensor assays for the detection of Lipoarabinomannan (and other biomarkers) from Mycobacteria and demonstrated feasibility in blinded clinical studies in humans and cattle, demonstrating one -health compatibility. We have also demonstrated detection of lipopolysacharides from eight different serotypes of Shiga-toxin carrying E. coli in beef, and Salmonella Typhimurium in pediatric patients using the same approach.

\section{Conclusions}

We have demonstrated clinical feasibility of a One Health Strategy for point-of-care diagnostics of bacterial infections in blood for tuberculosis and Gram-negative pathogens in clinical samples. The results will be demonstrated with several discussion points on the consideration of unconventional biochemistry of pathogens for diagnostics, factors influencing point-of-care deployment and integration of diagnostic platforms for biosurveillance. One Health Considerations and challenges therein will also merit discussion

\section{Keywords}

universal diagnostics; one health; deployable sensors

\section{Acknowledgments}

UBS concept and the Gram-positive and Gram-negative assay development was supported by Los Alamos National Laboratory Directed Research Award to McMahon and Mukundan. The Lipopolysacharide assay for cattle was supported by USDA (Mukundan). The Tuberculosis Diagnostics work was supported by NIH R21 (Dorman, JHU with Mukundan, LANL Lead). We thank several people at LANL, UNM, JHU for technical and administrative support. we thank the patients who generously contributed samples to our effort from Uganda, Kenya and South Korea.

\section{References}

Sakamuri, R; et al. Accurate Tracking of Bovine Tuberculosis Biomarkers in Infected Cattle using a Novel Biomarker Capture Strategy, Analytical Sciences, 33(4): 457-460, 2017.

Noormohammed A et al. Detection of Salmonella LPS in Patient blood by Lipoprotein Capture. Proc. SPIE 10072, Optical Diagnostics and Sensing XVII: Toward Point-of-Care Diagnostics, 100720A, 2017. doi: $10.1117 / 12.2253506$

Stromberg L et al. Membrane Insertion for the Detection of Lipopolysaccharides: Exploring the Dynamics of Amphiphile-inLipid Assays, PloS One, April 2016.

Sakamuri RM et al. Association of lipoarabinomannan with high density lipoprotein in blood: implications for diagnostics. Tuberculosis (Edinb). 2013 May;93(3):301-7. doi: 10.1016/j.tube.2013.02.015. Epub 2013 Mar 16

Mukundan $\mathbf{H}$ et al. Understanding the interaction of Lipoarabinomannan with membrane mimetic architectures. Tuberculosis, 92(1) 32-47, 2012.

Mukundan $\mathrm{H}$ et al., Waveguide-based Sensors for Pathogen Detection, Invited Review, Sensors, 9(7), 5783-5809.

\section{*Harshini Mukundan}

E-mail: HARSHINI@LANL.GOV 